\title{
Different scenarios of topological phase transitions in homogeneous neutron matter
}

\author{
S. S. Pankratov, ${ }^{1}$ M. Baldo, ${ }^{2}$ and M. V. Zverev ${ }^{1,3}$ \\ ${ }^{1}$ National Research Centre Kurchatov Institute, pl. Akademika Kurchatova 1, Moscow, 123182, Russia \\ ${ }^{2}$ Istituto Nazionale di Fisica Nucleare, Sezione di Catania, 64 Via S.-Sofia, I-95123 Catania, Italy \\ ${ }^{3}$ Moscow Institute of Physics and Technology, Institutskii per. 9, Dolgoprudnyi, Moscow region, 141700 Russia
}

(Dated: May 25, 2018)

\begin{abstract}
We study different scenarios of topological phase transitions in the vicinity of the $\pi^{0}$ condensation point in neutron matter. The transitions occur between the Fermi-liquid state and a topologically different one with two sheets of the Fermi surface. Two possibilities of a rearrangement of quasiparticle degrees of freedom are shown: the first-order topological phase transition and the second-order one. The order of the phase transition is found to be strongly dependent on the value of the critical wave vector of the soft $\pi^{0}$ mode. The thermodynamics of the system is also studied. It is shown that the topology of the quasiparticle momentum distribution is mainly determined by the neutron matter density, while the temperature $T$ is essential in a narrow density region. A simple explanation of the first-order topological phase transition at $T=0$ is given.
\end{abstract}

PACS numbers: 21.65.-f, 26.60.-c, 71.10.Ay

\section{INTRODUCTION}

Dense neutron matter is an example of systems in which correlations of single-particle degrees of freedom are strongly enhanced in a certain region of external parameters. Such enhancement is attributed to an exchange of critical fluctuations of a very soft collective mode in a vicinity of its collapse [1]. In dense neutron matter, critical spin-isospin fluctuations with quantum numbers of the neutral pion are enhanced close to the $\pi^{0}$ condensation point (PCP) [2]. Critical density for the neutral pion condensation $\rho_{c} \simeq 0.2 \mathrm{fm}^{-3}$ predicted in microscopic calculations $[3,4]$ is reached in a typical neutron star with the central density of $0.5-1.0 \mathrm{fm}^{-3}$.

Strong momentum dependence of the quasiparticle (QP) interaction due to exchange of critical spin-isospin fluctuations may result in a change in the topology of the ground state of a neutron QP system [5]. To our knowledge, the possibility of a change in the topology of the ground state was discussed for the first time in Ref. [6] for electronic systems. In nuclear physics, QP momentum distributions $n(\mathbf{p})$ with a topology different from that of the Fermi sphere $n_{\mathrm{FL}}(\mathbf{p})=\theta\left(p_{F}-p\right)$ were considered in Refs. [7, 8] for model interactions and in Ref. [9] for semirealistic ones.

The non-Fermi-liquid topology of the ground state QP momentum distribution in the vicinity of PCP was first discussed in Ref. [5]. With the increase of the density $\rho$ towards the PCP value $\rho_{c}$ the QP spectrum $\varepsilon(\mathbf{p})$ (measured from the chemical potential $\mu$ ) becomes a nonmonotonic function and at certain density $\rho_{b}$ touches the momentum axis at some point $p_{b}$ (see panel (a) of Fig. 1). This situation is associated with a quantum critical point (QCP) [10] at which the single-particle density of states diverges [11]. Beyond the QCP, the Landau state with the Fermi step QP distribution $n_{\mathrm{FL}}(\mathbf{p})$ becomes unstable
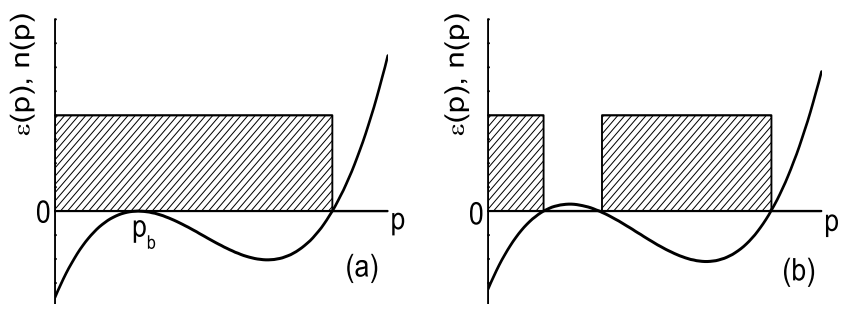

FIG. 1. Left panel: QCP at the $\rho_{b}$ density value. Right panel: Beyond the QCP.

as it violates the necessary stability condition

$$
\delta E[n]=2 \int \varepsilon(\mathbf{p},[n(\mathbf{p})]) \delta n(\mathbf{p}) d v>0 .
$$

Here $d v=d^{3} \mathbf{p} /(2 \pi)^{3}$ is the volume element in the momentum space and the factor 2 stands for summation over spin projections. The constraint (1) requires a positive change in the total energy $E[n]$ of the system for any admissible variation $\delta n(\mathbf{p})$ of the $\mathrm{QP}$ momentum distribution that conserves the density

$$
2 \int \delta n(\mathbf{p}) d v=0
$$

Thus a new state appears with an unoccupied region ("bubble") in the momentum distribution, which has several sheets of the Fermi surface (panel (b) of Fig. 1). This is the state that was considered in Refs. [7-9]. Further development concerning states with many bubbles can be found in Refs. [12, 13]. It is also worth noting investigations [14] concerning abnormal occupation in boson matter.

Reconstructions of the QP momentum distribution in strongly correlated Fermi systems changing the Fermi surface topology are generally referred [15] to as topological phase transitions (TPTs). It should be noted 
that, besides the bubble scenario, there is another type of TPT which is called the fermion condensation [16-18]. In this scenario, the QP spectrum acquires a flat band $\varepsilon(p)=0, p \in\left[p_{i}, p_{f}\right]$, and the Fermi surface changes its dimension. The relation between the bubble scenario and the fermion condensation is discussed in Ref. [19].

Going back to the neutron matter problem, we note that a bubble formation beyond the QCP is quite important for cooling of neutron stars [5, 20]. Indeed, a new sheet of the neutron Fermi surface with a low value of the corresponding Fermi momentum plays an important role for operation of the direct Urca processes: $n \rightarrow p+e^{-}+\overline{\nu_{e}}, p+e^{-} \rightarrow n+\nu_{e}$. In a neutron star's core these processes are generally considered to be forbidden [21] due to the kinematic restriction on the Fermi momenta of the involved particles, $p_{F_{n}} \leq p_{F_{p}}+p_{F_{e}}$. In the typical density range of the core $\sim 1-2 \rho_{0}$ ( $\rho_{0} \simeq 0.16 \mathrm{fm}^{-3}$ is the normal nuclear density), the proton fraction does not exceed $6-8 \%$, and the right-hand side of the kinematic equality is estimated by $\sim 0.8 p_{F_{n}}$. The appearance of the new sheet of the Fermi surface at the point $p_{F_{n}}^{(1)}<0.8 p_{F_{n}}$ provides agreement with the kinematic restriction. This mechanism was considered in Refs. [5, 20] as a possibility for the enhanced cooling of some neutron stars (e.g., Vela, Geminga, and 3C58).

\section{QUASIPARTICLE APPROACH NEAR PION CONDENSATION POINT}

\section{A. General relations}

The method we use for a description of neutron matter near the PCP is based on an implementation of the Landau-Migdal QP approach to strongly correlated Fermi systems that is reviewed in details in Ref. [22]. Within this approach, the QP spectrum and the QP momentum distribution at finite temperature $T$ are evaluated by solving the set of equations,

$$
\begin{aligned}
& \frac{\partial \varepsilon(\mathbf{p})}{\partial \mathbf{p}}=\frac{\mathbf{p}}{m}+\int f\left(\mathbf{p}, \mathbf{p}^{\prime}\right) \frac{\partial n\left(\mathbf{p}^{\prime}\right)}{\partial \mathbf{p}^{\prime}} d v^{\prime}, \\
& n(\mathbf{p})=\left[1+e^{\varepsilon(\mathbf{p}) / T}\right]^{-1}, \\
& 2 \int n(\mathbf{p}) d v=\rho .
\end{aligned}
$$

The first equation of this set is the Landau relation where $f\left(\mathbf{p}, \mathbf{p}^{\prime}\right)$ is the QP interaction function $[23,24]$ and $m$ stands for the free neutron mass; the second equation is the Fermi-Dirac formula in which $\varepsilon(\mathbf{p})$ is a functional of $n(\mathbf{p})$; and the last one is the normalization condition.

The QP interaction function is identified [23, 24] with the $\omega$ limit of the vertex function $\Gamma$,

$$
\begin{aligned}
f\left(\mathbf{p}_{1}, \boldsymbol{\sigma}_{1} ; \mathbf{p}_{2}, \boldsymbol{\sigma}_{2}\right)=Z^{2} \Gamma_{\alpha \beta, \gamma \delta}^{\omega}\left(\mathbf{p}_{1}, \mathbf{p}_{2}\right) \\
=Z^{2} \lim _{\frac{k}{\omega} \rightarrow 0, \omega \rightarrow 0} \Gamma_{\alpha \beta, \gamma \delta}\left(\mathbf{p}_{1}, \mathbf{p}_{2}, \mathbf{k}, \omega\right),
\end{aligned}
$$

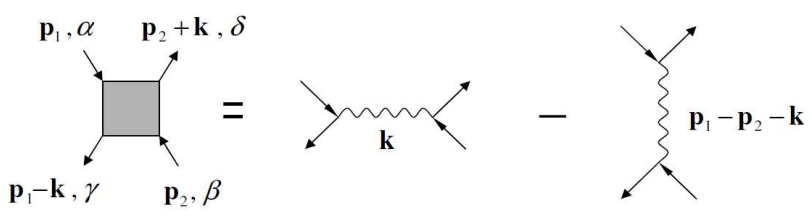

FIG. 2. The main contribution to the QP interaction function.

where $Z$ is the residue of the single-particle Green function and $\boldsymbol{\sigma}_{\alpha \gamma}$ stands for Pauli spin matrices. According to Ref. [1], the most singular contribution to the vertex function near the PCP comes from an exchange of a soft spin-isospin collective mode. The corresponding direct and the exchange graphs are shown in Fig. 2. At the limit $k \rightarrow 0$, the contribution of the exchange graph still exhibits a strong dependence on the relative momentum $\mathbf{q}=\mathbf{p}_{1}-\mathbf{p}_{2}$. Therefore the QP interaction function reads $[5]$

$$
f\left(\mathbf{p}_{1}, \boldsymbol{\sigma}_{1} ; \mathbf{p}_{2}, \boldsymbol{\sigma}_{2}\right) \simeq\left(\mathcal{J}_{\alpha \delta} D \mathcal{J}_{\beta \gamma}\right)(\mathbf{q}, \omega=0 ; \rho)
$$

where $\mathcal{J}_{\alpha \delta}$ is the interaction vertex of nucleons and pions in neutron media, $D$ is the $\pi^{0}$ propagator, and the general arguments of the operators are in parentheses.

\section{B. Topological phase transitions}

The occurrence of TPTs in dense neutron matter can be traced with the help of the strong momentum dependence of the QP interaction function near the PCP. As was discussed in Ref. [2], the spectrum $\omega(q)$ of $\pi^{0}$-like collective excitations in neutron matter is given by a particular branch of poles of the $\pi^{0}$ propagator. The behavior of this branch depends on the density $\rho$ of the system. Pion condensation occurs at the critical density $\rho_{c}$ where the excitation energy vanishes, $\omega\left(q_{c}\right)=0$, together with its derivative, $\partial \omega\left(q_{c}\right) / \partial q=0$, at a certain momentum $q_{c}$. As a consequence, the following conditions at the PCP are valid:

$$
D^{-1}\left(q_{c}, 0 ; \rho_{c}\right)=0,\left.\quad \frac{\partial D^{-1}\left(q, 0 ; \rho_{c}\right)}{\partial q^{2}}\right|_{q_{c}}=0 .
$$

Thus using the Taylor expansion of $D^{-1}$, the interaction function $f$ entering in Eq. (3) can be written [5, 20, 25] in the form

$$
\begin{aligned}
f\left(\mathbf{p}_{1}, \mathbf{p}_{2}\right)=\frac{1}{2} \operatorname{Tr}_{\boldsymbol{\sigma}_{1}} \operatorname{Tr}_{\boldsymbol{\sigma}_{2}} f\left(\mathbf{p}_{1}, \boldsymbol{\sigma}_{1} ; \mathbf{p}_{2}, \boldsymbol{\sigma}_{2}\right) \\
=\frac{g}{\kappa^{2}(\rho)+\left(\left(\mathbf{p}_{1}-\mathbf{p}_{2}\right)^{2} / q_{c}^{2}-1\right)^{2}}
\end{aligned}
$$

where $g$ is an effective coupling constant and $\kappa^{2}(\rho) \propto$ $\left(\rho_{c}-\rho\right)$ is an effective radius in momentum space. The notation $\operatorname{Tr}_{\boldsymbol{\sigma}}$ stands for the trace over the spin variable. 


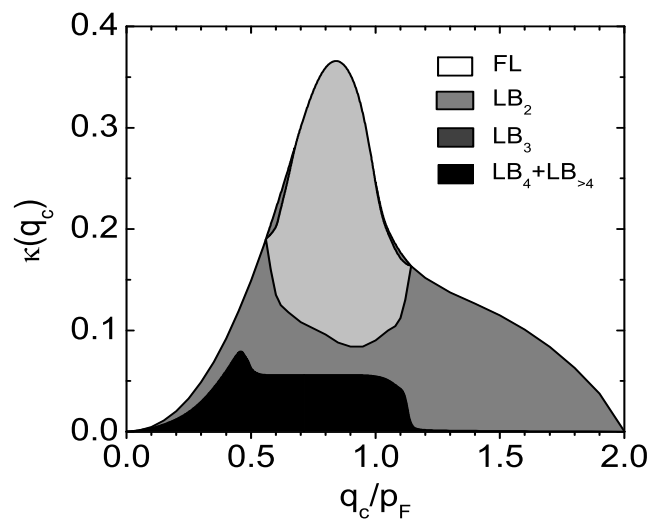

FIG. 3. The phase diagram of the neutron matter ground state near the PCP [25].

Previous investigations [25] within the QP interaction (9) were focused on the ground-state topology, and $g, q_{c}, \kappa$ quantities were regarded as phenomenological parameters. For convenience of readers, we present in Fig. 3 a topological phase diagram in $q_{c}, \kappa$ variables obtained in that work. The label FL corresponds to the Fermi-liquid state and $\mathrm{LB}_{i}$, to states with $i$ sheets of the Fermi surface. A change in the density $\rho$ leads to a change in the system position $\left(q_{c}, \kappa\right)$ on the diagram. Transitions between different regions of the phase diagram represent TPTs that can occur in neutron matter. Until recently, all such TPTs were considered $[19,22]$ to occur continuously according to violation of Eq. (1). However, an attentive investigation [26] within the model (9) revealed another possibility, namely, a first-order TPT. Such a scenario of the Fermi surface reconstruction in a homogeneous isotropic Fermi system was first found [27] in a model of strongly correlated 2D electron gas beyond the QCP. Below, we present a detailed analysis of possible TPTs for the neutron matter problem.

\section{SEMI-MICROSCOPIC QP INTERACTION FUNCTION}

The above discussion of TPTs was restricted by the phenomenological description (9) of the QP interaction function. It is possible to convey neutron matter physics near the PCP in a more direct way. A semi-microscopical expression for the QP interaction can be derived by use of the microscopic formula (7).

The bare $\pi^{0}$-nucleon interaction vertex is given [28] by

$$
J_{\pi N N}^{0}=\frac{i f}{m_{\pi}}(\boldsymbol{\sigma} \mathbf{q}) \tau_{3}
$$

where $f \simeq 1$ is the dimensionless $\pi^{0}$-nucleon coupling constant, $m_{\pi}$ is the pion mass, and $\tau_{3}$ is the diagonal isospin matrix. The vertex renormalization in neutron matter is due to nucleon-nucleon correlations that can

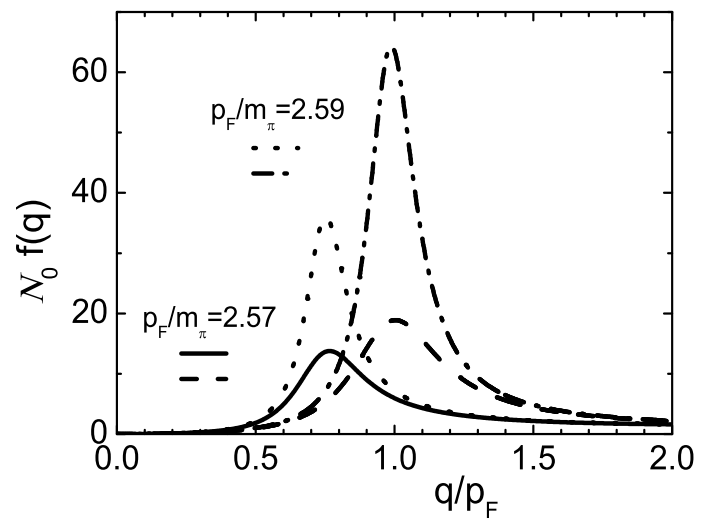

FIG. 4. The interaction function (15) multiplied by $\mathcal{N}_{0}=m p_{F} / \pi^{2}$ as a function of $q / p_{F}$. The parametrization (16) corresponds to the curves peaked at $q_{c} \simeq$ $0.74 p_{F}$, while (17) corresponds to $q_{c} \simeq p_{F}$.

be described by means of the Landau-Migdal amplitude $[29]$

$$
\frac{m^{*} p_{0}}{\pi^{2}} \mathcal{F}=F+F^{\prime} \boldsymbol{\tau}_{1} \boldsymbol{\tau}_{2}+\left(G+G^{\prime} \boldsymbol{\tau}_{1} \boldsymbol{\tau}_{2}\right) \boldsymbol{\sigma}_{1} \boldsymbol{\sigma}_{2}
$$

Here $p_{0}=\left(1.5 \pi^{2} \rho_{0}\right)^{1 / 3}$ is the Fermi momentum in equilibrium nuclear matter and $m^{*}$ is the effective nucleon mass. According to Ref. [2], the renormalized static vertex reads

$$
J_{\alpha \beta}^{s t}(\mathbf{q})=\frac{i f}{m_{\pi}} \frac{\left(\boldsymbol{\sigma}_{\alpha \beta} \mathbf{q}\right) \tau_{3}}{1+g_{c} \chi_{N N}^{s t}(q)}, \quad g_{c}=\frac{\pi^{2}}{m^{*} p_{0}} \frac{m_{\pi}^{2}}{f^{2}}\left(G+G^{\prime}\right) .
$$

The function $\chi_{N N}^{s t}(q)$ is the static susceptibility of free QPs proportional to the Lindhard function:

$$
\begin{aligned}
\chi_{N N}^{s t}(q) & =\frac{f^{2}}{m_{\pi}^{2}} \frac{m^{*} p_{F}}{\pi^{2}} \\
\times & \left(\frac{1}{2}+\frac{p_{F}}{2 q}\left(\frac{q^{2}}{4 p_{F}^{2}}-1\right) \ln \left|\frac{1-q / 2 p_{F}}{1+q / 2 p_{F}}\right|\right),
\end{aligned}
$$

where $p_{F}=\left(3 \pi^{2} \rho\right)^{1 / 3}$ is the neutron Fermi momentum.

The pion propagator in Eq. (7) is connected with the polarization operator: $D^{-1}(q, \omega)=\omega^{2}-\mathbf{q}^{2}-m_{\pi}^{2}-$ $\Pi(q, \omega)$. The microscopic description of the pion polarization operator is a quite subtle matter [28]. We use here its semi-microscopic representation [30]

$$
\Pi(q, 0)=-q^{2}\left(\frac{\chi_{N N}^{s t}(q)}{1+g_{c} \chi_{N N}^{s t}(q)}+\frac{\rho}{\rho_{\Delta}\left(1+q^{2} / q_{\Delta}^{2}\right)}\right) .
$$

The first term describes processes of particle-hole excitations where nucleon-nucleon correlations are taken into account by the denominator. The second term is a phenomenological one and describes $\Delta$-resonance-hole excitations. The $S$-scattering processes in neutron matter are neglected. 
Finally, one arrives at the formula

$$
\begin{aligned}
f(q) & =\frac{1}{2} \operatorname{Tr}_{\boldsymbol{\sigma}_{1}} \operatorname{Tr}_{\boldsymbol{\sigma}_{2}} f\left(\mathbf{p}_{1}, \boldsymbol{\sigma}_{1} ; \mathbf{p}_{2}, \boldsymbol{\sigma}_{2}\right) \\
= & \left(\frac{f}{m_{\pi}} \frac{q}{1+g_{c} \chi_{N N}^{s t}(q)}\right)^{2} \frac{1}{m_{\pi}^{2}+q^{2}+\Pi(q, 0)}
\end{aligned}
$$

for the interaction function, where the polarization operator is given by (14). In Eq. (15), the constants $f=1$, $m^{*}=m, G+G^{\prime}=1$ are fixed all along the further discussion, while two sets of parameters for the phenomenological part of the polarization operator are used. The first set,

$$
\rho_{\Delta}=0.59 m_{\pi}^{3}, \quad q_{\Delta}=2.08 m_{\pi},
$$

corresponds to Ref. [30], while the second one,

$$
\rho_{\Delta}=0.97 m_{\pi}^{3}, \quad q_{\Delta}=4.1 m_{\pi},
$$

is suggested in this work. Both sets reproduce the value $\rho_{c} \simeq 0.2 \mathrm{fm}^{-3}$ that agrees with the critical density of $\pi^{0}$ condensation obtained in $[3,4]$. The difference between the two parametrizations is in the corresponding value of the critical wave vector $q_{c}$, which is not known accurately from microscopic calculations. We also note that $\rho_{c}$ and $q_{c}$ are quite sensitive to tuning of parameters $\rho_{\Delta}$ and $q_{\Delta}$. The behavior of the QP interaction function is shown in Fig. 4. The parametrization (16) leads to $q_{c} \simeq 0.74 p_{F}$ (that is less then $\left.p_{F}\right)$, while (17) leads to $q_{c} \simeq p_{F}$. Figure 4 also demonstrates the amplification of the QP interaction with an increase of $p_{F}$ (equally, the density $\left.\rho=p_{F}^{3} / 3 \pi^{2}\right)$. The divergence is reached at the PCP point, $p_{F c} \simeq 2.602 m_{\pi}$ and $p_{F c} \simeq 2.598 m_{\pi}$ correspondingly for the first and the second parametrizations.

\section{DIFFERENT SCENARIOS OF TOPOLOGICAL PHASE TRANSITIONS}

In this Section, we discuss results of the analysis of topological rearrangements of QP degrees of freedom based on the semi-microscopic QP interaction function. The QP spectrum and the QP momentum distribution are determined by the set of Eqs. (3),(4),(5). Due to the assumed dependence of the interaction function (15) on the relative momentum $q$, Eq. (3) can be integrated by parts, yielding

$$
\varepsilon(\mathbf{p})=\frac{p^{2}}{2 m}-\mu+\int f\left(\mathbf{p}-\mathbf{p}^{\prime}\right) n\left(\mathbf{p}^{\prime}\right) d v^{\prime} .
$$

For solving the equations a contracting iterations method was used. The case of $T=0$ was modeled by $T=$ $10^{-5} \varepsilon_{F}^{0}$, where $\varepsilon_{F}^{0}=p_{F}^{2} / 2 m$.

First, we consider the results obtained within the model (16) where $q_{c}=0.74 p_{F}$. The evolution of QP degrees of freedom, driven by an increase of $p_{F}$, is displayed in Fig. 5. It has a continuous behavior in agreement with
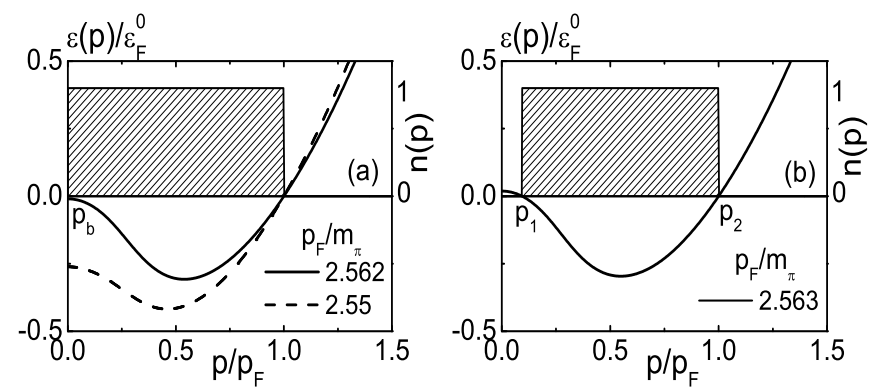

FIG. 5. Evolution of the QP spectrum (in units $\varepsilon_{F}^{0}=$ $\left.p_{F}^{2} / 2 m\right)$ and the QP momentum distribution with increasing $p_{F}$ within the model (16). The temperature $T=0$.

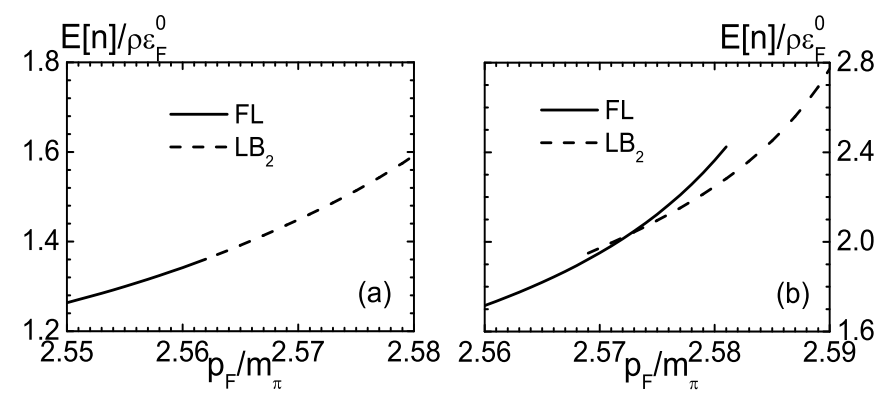

FIG. 6. Energy per particle at $T=0$ as a function of $p_{F}$. The left panel corresponds to the model (16), while right one corresponds to the model (17).

the scenario of TPTs with violation of the necessary stability condition (1). As the Fermi momentum reaches the critical value $p_{F}^{b} \simeq 2.562 m_{\pi}$, a bifurcation occurs, and a new zero of the QP spectrum $\varepsilon(p)$ appears at the momentum $p_{b}=0$. Beyond the $p_{F}^{b}$, the QP momentum distribution possesses two sheets of the Fermi surface with coordinates $p_{1} \ll p_{F}$ and $p_{2} \simeq p_{F}$. The size of the bubble region (which is equal to $p_{1}$ ) increases continuously from the zero value with further increase of $p_{F}$.

Dealing with Eq. (18) for the QP spectrum, one can consider the corresponding energy functional

$$
E[n]=2 \int \frac{p^{2}}{2 m} n(\mathbf{p}) d v+\int f\left(\mathbf{p}-\mathbf{p}^{\prime}\right) n(\mathbf{p}) n\left(\mathbf{p}^{\prime}\right) d v d v^{\prime} .
$$

The behavior of the energy is given in panel (a) of Fig. 6. It shows a monotonic increase according to the continuous picture of the evolution of the ground state in Fig. 5. A second-order topological transition occurs between the FL and the $\mathrm{LB}_{2}$ states exactly at the critical point $p_{F}^{b} \simeq 2.562 m_{\pi}$.

Passing to the model (17) where $q_{c} \simeq p_{F}$, we first consider panel (b) of Fig. 6. As it is seen, there are two different states in the interval $2.57 m_{\pi} \lesssim p_{F} \lesssim 2.58 m_{\pi}$. The coexistence of several solutions of Eqs. (4),(5),(18) was established by considering different initial conditions 


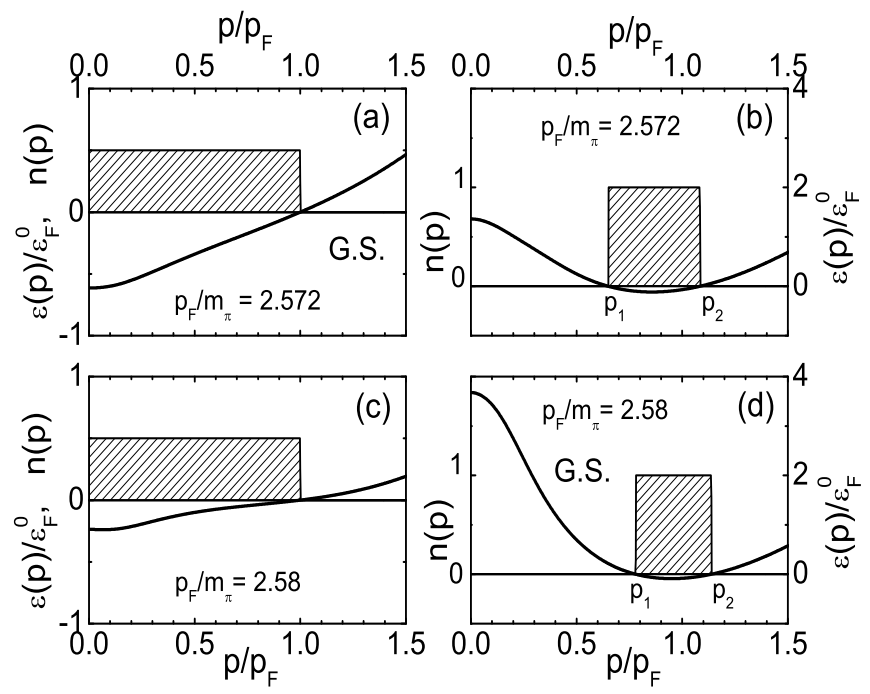

FIG. 7. Panels (a) and (b): QP spectra and QP momentum distributions of coexisting states in advance of the first-order TPT. Panels (c) and (d): The same beyond the TPT point. The temperature $T=0$.

for the iteration procedure. Fig. 7 represents QP spectra and QP momentum distributions of coexisting states. At the value $p_{F}=2.572 m_{\pi}$ the ground state is the FL one (panel (a)), while there is also a $\mathrm{LB}_{2}$ state (panel (b)) with a slightly higher energy value. It is worth emphasizing that the bubble region has finite, not negligible size $p_{1} \simeq 0.6 p_{F}$. A first-order phase transition occurs at $p_{F}^{1 s t} \simeq 2.573 m_{\pi}$. Beyond the transition point, the $\mathrm{LB}_{2}$ state (panel (d)) becomes energetically favored over the FL state (panel (c)). Finally, the local energy minimum of the functional (19), corresponding to the FL state, becomes unstable [31] (for $p_{F} \gtrsim 2.581 m_{\pi}$ ) and only the $\mathrm{LB}_{2}$ ground state remains (see panel (b) of Fig. 6).

We proceed with a thermodynamic analysis concerning the case of the first-order TPT. Thermodynamic functions of neutron matter with the Fermi momentum $p_{F}=2.572 m_{\pi}$ are given by Fig. 8. Panel (a) demonstrates that the FL state is lower in the energy than the $\mathrm{LB}_{2}$ one up to the temperature $T \sim 0.1 \varepsilon_{F}^{0} \sim 5 \mathrm{MeV}$. At the same time, panel (b) shows that the entropy

$$
S[n]=-2 \int n(\mathbf{p}) \ln n(\mathbf{p})+(1-n(\mathbf{p})) \ln (1-n(\mathbf{p})) d v,
$$

of the $\mathrm{LB}_{2}$ state grows more rapidly with increasing temperature than the entropy of the FL state. An interplay of contributions to the free energy $F[n]=E[n]-T S[n]$ leads to a first-order TPT driven by the temperature. Behavior of the free energy given by panel (c) demonstrates the first-order phase transition at the temperature $T_{1} \simeq 6.2 \cdot 10^{-2} \varepsilon_{F}^{0}$. Finally, panel $(\mathrm{d})$ shows the temperature evolution of the density of states

$$
\mathcal{N}[n]=\int \frac{d n(\mathbf{p})}{d \varepsilon} d v=\frac{1}{T} \int n(\mathbf{p})(1-n(\mathbf{p})) d v .
$$

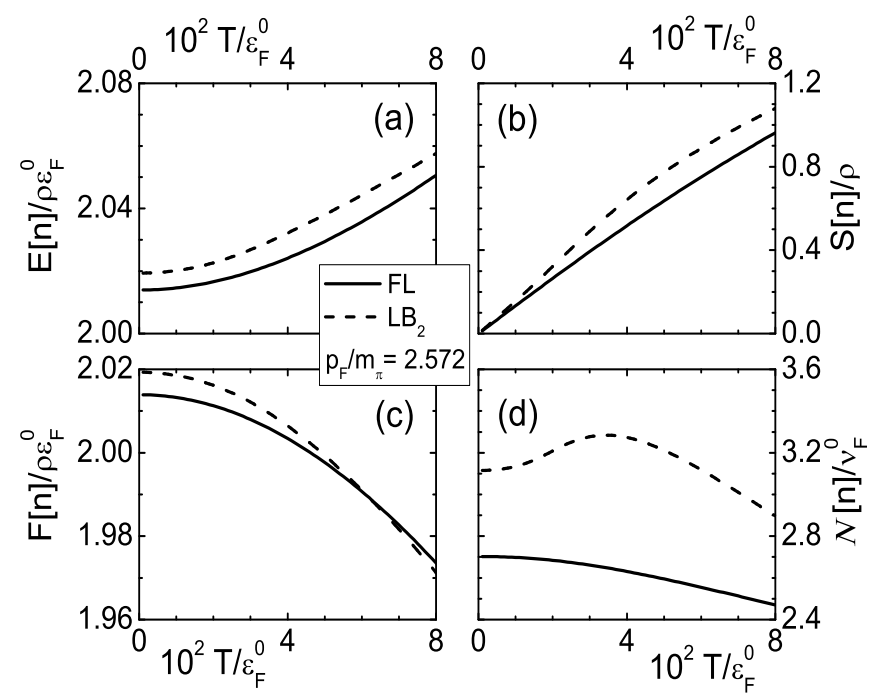

FIG. 8. The energy (a), the entropy (b), the free energy (c) and the density of states (d) as functions of the temperature for the FL and the $\mathrm{LB}_{2}$ states.
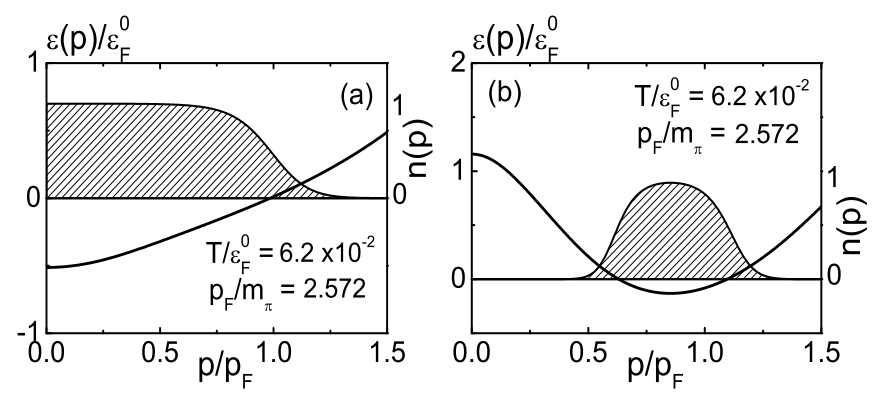

FIG. 9. QP spectra and momentum distributions of the $\mathrm{FL}$ and the $\mathrm{LB}_{2}$ states at the point (the black dot $\mathrm{A}$ in Fig. 10 below) of the temperature-driven TPT.

To elucidate the temperature behavior of the thermodynamic functions under consideration, we present in Fig. 9 QP spectra and QP momentum distributions of the FL state and of the $\mathrm{LB}_{2}$ one at the point $T=6.2 \cdot 10^{-2} \varepsilon_{F}^{0}$ near the phase transition. This figure shows that the entropy and the density of states of the $\mathrm{LB}_{2}$ state are larger than those of the FL state due to the fact that in the first case, two sheets of the Fermi surface "melt", while in the second case only one does.

The results of the analysis on the temperature behavior of QP states, in the region near the point $p_{F}=2.572 m_{\pi}$ (see panel (b) of Fig. 6), are summarized by the $\left(p_{F}, T\right)$ phase diagram of neutron matter shown in Fig. 10. The diagram consists of two regions: the first one corresponds to the FL phase of the system, while the other one to the $\mathrm{LB}_{2}$ phase. The regions are separated by the line $T_{1}\left(p_{F}\right)$ of the first-order TPTs. The diagram shows that neutron matter state topology is determined by the temperature in a quite narrow density interval. 


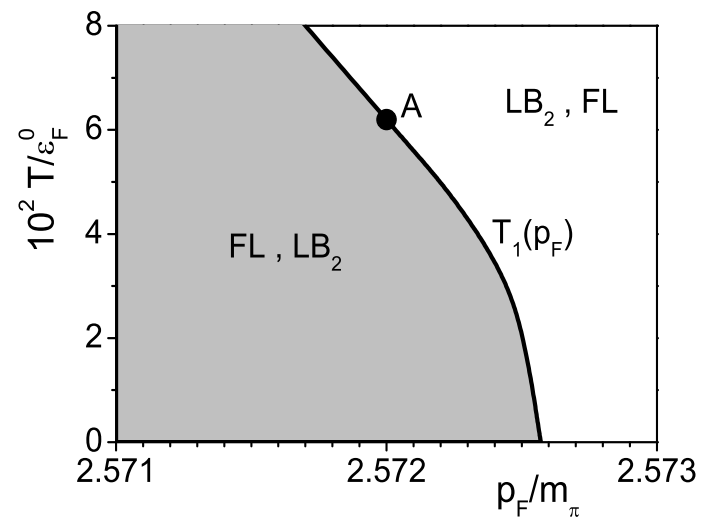

FIG. 10. Phase diagram of neutron matter near the line $T_{1}\left(p_{F}\right)$ of the fist-order TPTs. The first label corresponds to the thermodynamically favored phase, while the second one corresponds to a metastable phase. The black dot A refers to the QP states shown in Fig. 9.

\section{ENERGETICS OF LB $_{2}$ STATES}

In this Section, we elucidate why the system appears to be in the $\mathrm{LB}_{2}$ phase. The energy functional (19) can be rewritten in the form

$$
E[n]=2 \int \frac{p^{2}}{2 m} n(\mathbf{p}) d v+\frac{1}{2} \int f(\mathbf{q}) S(\mathbf{q} ;[n]) d v .
$$

Here the interaction energy is given by means of the structure function

$$
S(\mathbf{q} ;[n])=\frac{2}{\rho} \int n(\mathbf{p}+\mathbf{q}) n(\mathbf{p}) d v .
$$

In the case $T=0$, which we address below, the set of all possible QP momentum distributions with one or two sheets of the Fermi surface (that is the FL or $\mathrm{LB}_{2}$ states) is specified by

$$
\begin{gathered}
n_{2}(\mathbf{p})=\theta\left(p_{2}-p\right)-\theta\left(p_{1}-p\right), \\
p_{2}^{3}-p_{1}^{3}=p_{F}^{3} .
\end{gathered}
$$

The last equation follows from the normalization condition (5). This class is referred below as QP distributions of $n_{2}$ type. In this case, the structure function is evaluated explicitly, the corresponding formulas are given in the Appendix. It is sufficient, due to Eq. (25), to deal with one parameter. The convenient choice is $\eta=p_{2}-p_{1}$ (the width of the occupied region in the QP momentum distribution) that defines distribution parameters

$$
p_{1,2}=\frac{1}{\sqrt{3}} \sqrt{\frac{p_{F}^{3}}{\eta}-\left(\frac{\eta}{2}\right)^{2}} \mp \frac{\eta}{2} .
$$

The value $\eta=p_{F}$ corresponds to $p_{1}=0, p_{2}=p_{F}$ i.e. to the case of the Fermi step. The decrease of $\eta$ leads to a monotonic increase of $p_{1,2}$.

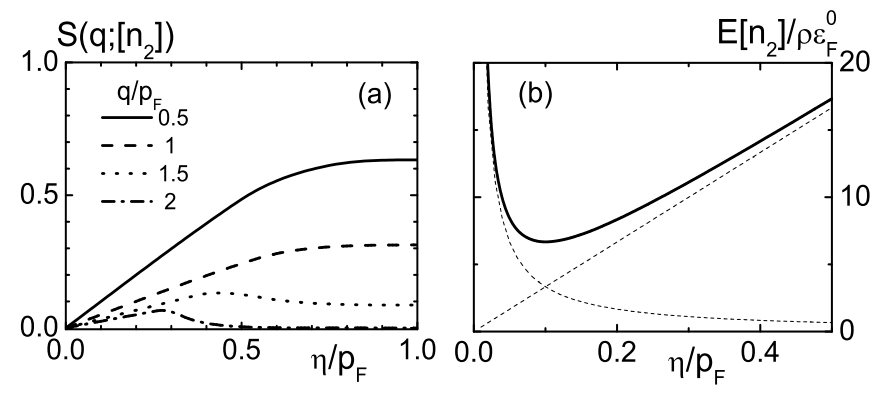

FIG. 11. Left panel: The structure function for QP momentum distributions of the $n_{2}$ type versus the ratio $\eta / p_{F}$ at different values of the wave vector $q$. Right panel: The total energy per particle for the thin $n_{2}$ type momentum distributions.

The behavior of the structure function $S\left(\mathbf{q} ;\left[n_{2}\right]\right)=$ $S(q ; \eta)$ is shown in panel (a) of Fig. 11.

Its explicit form, corresponding to the Landau state $\left(\eta=p_{F}\right)$, is well known

$$
\begin{aligned}
S\left(q ; p_{F}\right) & =S_{\mathrm{FL}}(q) \\
= & \frac{1}{2}\left(1-\frac{q}{2 p_{F}}\right)^{2}\left(2+\frac{q}{2 p_{F}}\right) \theta\left(2 p_{F}-q\right) .
\end{aligned}
$$

It is remarkable that in the other important case of thin $\left(\eta \ll p_{F}\right) n_{2}$-type QP distributions that can appear by a first-order TPT, one obtains

$$
\left.S(q ; \eta)\right|_{\eta \rightarrow 0}=\frac{\eta}{2 q}
$$

This result is demonstrated well by Fig. 11, and reflects a decrease of the overlap of the momentum distributions in the integral in Eq. (23). As a consequence, this leads to a reduction of the interaction energy,

$$
\left.E_{i n t}\left[n_{2}\right]\right|_{\eta \ll p_{F}} \simeq \frac{U \eta}{2 p_{F}}, \quad U=p_{F} \int \frac{f(q)}{2 q} d v .
$$

The kinetic energy, on the contrary, shows enhancement in agreement with the explicit result

$$
\begin{aligned}
E_{k i n}\left[n_{2}\right]=\frac{3}{5 p_{F}^{5}}( & \left.p_{2}^{5}-p_{1}^{5}\right) \varepsilon_{F}^{0} \rho \\
& =\left(\frac{p_{F}}{3 \eta}+\frac{\eta^{2}}{3 p_{F}^{2}}-\frac{\eta^{5}}{15 p_{F}^{5}}\right) \varepsilon_{F}^{0} \rho .
\end{aligned}
$$

The total energy in the case of distributions of the $n_{2}$ type in the limit $\eta \ll p_{F}$ is given by

$$
E\left[n_{2}\right] \simeq\left(\frac{p_{F}}{3 \eta}+\frac{u \eta}{2 p_{F}}\right) \varepsilon_{F}^{0} \rho, \quad u=U / \varepsilon_{F}^{0} \rho .
$$

This function is plotted on panel (b) of Fig. 11 and shows a minimum value

$$
E_{\mathrm{LB}_{2}}=\sqrt{\frac{2}{3} u} \varepsilon_{F}^{0} \rho
$$


at the point $\eta_{c}=p_{F} \sqrt{2 /(3 u)}$. Finally, we note that the $E_{\mathrm{LB}_{2}}$ energy appears to be lower than the Fermi-liquid one,

$$
E_{\mathrm{FL}}=\frac{3}{5} \varepsilon_{F}^{0} \rho+\frac{1}{2} \int f(q) S_{\mathrm{FL}}(q) d v,
$$

when the QP interaction is sufficiently strong. Indeed, if one characterizes the $\mathrm{QP}$ interaction function by an effective coupling constant $f(q) \propto g$, Eqs. (32) and (33) show that $E_{\mathrm{LB}_{2}} \propto \sqrt{g}$ and $E_{\mathrm{FL}} \propto g$ at the large- $g$ limit.

Thus, the appearance of $\mathrm{LB}_{2}$ states is explained by the interplay between the kinetic and the interaction energy contributions. The existence of this energy minimum, generally, does not dependent on whether the Landau state is present or not. We remark that this simple explanation is limited to regarding the $n_{2}$ set of QP momentum distributions. A generalization to a more complete class $n_{\alpha>2}$ could in principle reveal an instability of a $\mathrm{LB}_{2}$ state with respect to some energetically favored one [26]. The solid proof of the existence of the $\mathrm{LB}_{2}$ ground state comes from the direct solution of Eqs. (4),(5), and (18).

\section{CONCLUSION}

In this article we have considered two scenarios of topological phase transitions in homogeneous neutron matter. The transitions occur between the Fermi-liquid state and the other one with two sheets of the Fermi surface. The investigation was performed with the use of a semimicroscopic expression for the quasiparticle interaction function in the vicinity of $\pi^{0}$ condensation point. The order of the phase transition is shown to depend on the value of the critical wave vector $q_{c}$. The first possibility for a rearrangement of the quasiparticle degrees of freedom is the second-order topological phase transition. It occurs when $q_{c}<p_{F}$ and corresponds to a quantumcritical-point scenario $[10,11,19]$ of the Fermi surface reconstruction. The second possibility studied in this work is the first-order topological phase transition. This case occurs when $q_{c} \gtrsim p_{F}$ and is connected with a sudden change in the quasiparticle momentum distribution and spectrum. The first-order topological phase transition can be driven by change of either the density or the temperature. Thermodynamic functions and the phase diagram have been calculated. It is shown that the influ- ence of the temperature on the Fermi surface topology is essential in a quite narrow density region. A simple explanation of the origin of the first-order topological phase transition at $T=0$ is given.

\section{ACKNOWLEDGMENTS}

We thank V. A. Khodel and E. E. Saperstein for their interest to this work and useful discussions. One of the authors (S.S.P.) would like to thank INFN (Sezione di Catania) for hospitality during his stay in Catania. This research was partially supported by Grants No. NSh7235.2010.2 and No. 2.1.1/4540 of the Russian Ministry for Science and Education, and by the RFBR Grants No. 11-02-00467-a and No. 12-02-00955-a.

\section{APPENDIX}

The structure function within the $n_{2}$ set of QP momentum distributions reads

$$
S\left(\mathbf{q} ;\left[n_{2}\right]\right)=\frac{2}{\rho} \int n(\mathbf{p}+\mathbf{q}) n(\mathbf{p}) d v=S_{11}+S_{22}-2 S_{12} .
$$

Here $(4 \pi / 3) p_{F}^{3} S_{i j}$ is a volume the intersection of two spheres with radii $p_{\min }=p_{i}$ and $p_{\max }=p_{j}, p_{\min } \leq p_{\max }$, while the distance between their centers is equal to the $\mathbf{q}$ vector length. The value of the volume is specified by the expression

$$
\begin{aligned}
& \quad S_{i j}\left(q ; p_{i}, p_{j}\right)=\left(\zeta\left(q ; p_{i}, p_{j}\right)+\zeta\left(q ; p_{j}, p_{i}\right)\right) \\
& \times \theta\left(p_{i}+p_{j}-q\right) \theta\left(q+p_{i}-p_{j}\right)+p_{i}^{3} \theta\left(p_{j}-q-p_{i}\right) .
\end{aligned}
$$

The first term corresponds to an intersection case, while the second one corresponds to a complete enclosure of the smaller sphere into the bigger one. The function $\zeta$ has the form

$$
\begin{aligned}
\zeta\left(q ; p_{i}, p_{j}\right)=\frac{1}{4}\left(p_{i}-\right. & \left.\frac{p_{i}^{2}+q^{2}-p_{j}^{2}}{2 q}\right)^{2} \\
& \times\left(2 p_{i}+\frac{p_{i}^{2}+q^{2}-p_{j}^{2}}{2 q}\right) .
\end{aligned}
$$

[1] A. M. Dyugaev, Sov. Phys. JETP, 43, 1247 (1976).

[2] A. B. Migdal, Rev. Mod. Phys. 50, 107 (1978).

[3] R. B. Wiringa, V. Fiks and A. Fabrocini, Phys. Rev. C 38, 1010 (1988).

[4] A. Akmal, V. R. Pandharipande and D. G. Ravenhall, Phys. Rev. C 58, 1804 (1998) [arXiv:nucl-th/9804027]; A. Akmal, V. R. Pandharipande, ibid. 56, 2261 (1997) [arXiv:nucl-th/9705013].
[5] D. N. Voskresensky, V. A. Khodel, M. V. Zverev and J. W. Clark, The Astrophysical Journal 533, 127 (2000) [arXiv:astro-ph/0003172].

[6] H. Fröhlich, Phys. Rev. 79, 845 (1950).

[7] M. de Llano, J. P. Vary, Phys. Rev. C 19, 1083 (1979).

[8] M. de Llano, A. Plastino, J. G. Zabolitzky, Phys. Rev. C 20, 2418 (1979). 
[9] V. C. Aguilera-Navarro, R. Belehrad, M. de Llano, M. Sandel, J. P. Vary, O. Rojo, Phys. Rev. C 22, 1260 (1980).

[10] V. A. Khodel, JETP Lett. 86, 721 (2008) [arXiv:0709.3653].

[11] V. A. Khodel, J. W. Clark, M. V. Zverev, JETP Lett. 94, 73 (2011) [ arXiv:1105.2552].

[12] M. V. Zverev and M. Baldo, JETP 87, 1129 (1998) [arXiv:cond-mat/9807324].

[13] S. A. Artamonov, Yu. G. Pogorelov, V. R. Shaginyan, JETP Lett. 68, 942 (1998) [arXiv:cond-mat/9811024].

[14] V. C. Aguilera-Navarro, R. Barrera, M. de Llano, J. W. Clark, A. Plastino, Phys. Rev. C 25, 560 (1982).

[15] G. E. Volovik, Quantum Phase Transitions from Topology in Momentum Space, Springer Lecture Notes in Physics Vol. 718 (Springer, Berlin, 2007), p. 31.

[16] V. A. Khodel and V. R. Shaginyan, JETP Lett. 51, 553 (1990).

[17] G. E. Volovik, JETP Lett. 53, 222 (1991).

[18] P. Nozières, J. Phys. I (France) 2, 443 (1992).

[19] V. A. Khodel, J. W. Clark, M. V. Zverev, Phys. Rev. B 78, 075120 (2008) [arXiv:0806.1908].

[20] V. A. Khodel, J. W. Clark, M. Takano and M. V. Zverev, Phys. Rev. Lett. 93, 151101 (2004).

[21] P. Haensel, A. Y. Potekhin, D. G. Yakovlev, Neutron Stars 1, Equation of State and Structure, Springer As- trophysics and Space Science Library Vol. 326 (Springer, Berlin, 2007).

[22] V. A. Khodel, J. W. Clark, M. V. Zverev, Phys. Atom. Nucl. 74, 1237 (2011) [arXiv:1108.4023].

[23] E. M. Lifshitz, L. P. Pitaevsky, L. D. Landau and E. M. Lifshitz Course of Theoretical Physics Vol. 9, Statistical Physics Part 2 (Pergamon Press, Oxford, 1980).

[24] A. A. Abrikosov, L. P. Gor'kov, I. E. Dzyaloshinski, Methods of Quantum Field Theory in Statistical Physics (Prentice-Hall, London, 1963).

[25] M. Baldo, V. V. Borisov, J. W. Clark, V. A. Khodel and M. V. Zverev, J. Phys.: Condens. Matter 16, 6431 (2004) [arXiv:cond-mat/0402481].

[26] S. S. Pankartov, M. V. Zverev, JETP Lett., to be published.

[27] S. S. Pankartov, M. V. Zverev, M. Baldo, JETP Lett. 93, 591 (2011) [arXiv:1106.5160].

[28] T. Ericson, W. Weise, Pions and Nuclei (Claredon Press, London, 1988).

[29] A. B. Migdal, Theory of Finite Fermi Systems and Applications to Atomic Nuclei (Wiley, New York, 1967).

[30] A. B. Migdal, O. A. Markin, and I. I. Mishustin, Sov. Phys. JETP, 39, 212 (1974).

[31] Detailed analysis of the energy functional (19) with a QP interaction amplitude of the form (9) can be found in Ref. [26] for nuclear matter and in Ref. [27] for 2D electron gas problems. 